\title{
Performance Analysis of Group-Synchronized DCF for Dense IEEE 802.11 Networks
}

\author{
Lei Zheng, Student Member, IEEE, Minming Ni, Member, IEEE, Lin Cai, Senior Member, IEEE, \\ Jianping Pan, Senior Member, IEEE, Chittabrata Ghosh, and Klaus Doppler
}

\begin{abstract}
In dense IEEE 802.11 networks, improving the efficiency of contention-based media access control is an important and challenging issue. Recently, the IEEE802.11ah Task Group has discussed a group-synchronized distributed coordination function (GS-DCF) for densely deployed wireless networks with a large number of stations. By using the restricted access window (RAW) and RAW slots, the GS-DCF is anticipated to improve the throughput substantially, primarily due to relieving the channel contention. However, optimizing the MAC configurations for the RAW, i.e., the number and duration of RAW slots, is still an open issue. In this paper, we first build an analytical model to track the performance of the GS-DCF in saturated 802.11 networks. Then, we study and compare the GS-DCF throughput using both centralized and decentralized grouping schemes. The accuracy of our model has been validated with simulation results. It is observed that the GS-DCF obtains a throughput gain of seven times or more over DCF in a network of 512 or more stations. Moreover, it is demonstrated that the decentralized grouping scheme can be implemented with a small throughput loss when compared with the centralized grouping scheme.
\end{abstract}

Index Terms-Group-synchronized distributed coordination function (GS-DCF), restricted access window (RAW), EDCA, centralized/decentralized grouping scheme, saturated throughput, dense networks, IEEE 802.11ah.

\section{INTRODUCTION}

I $\mathrm{N}$ recent years, we have witnessed the explosive growth in the number of wireless devices and their possible applications around the world. ${ }^{1}$ For example, in the emerging area of machine-to-machine (M2M) communications, a large number

Manuscript received November 4, 2013; revised April 7, 2014; accepted June 22, 2014. Date of publication July 9, 2014; date of current version November 7, 2014. This paper was presented in part at the IEEE GLOBECOM 2013, Atlanta, GA, USA. This work was supported by the Natural Sciences and Engineering Research Council (NSERC) of Canada and in part by the Nokia Research Center, Berkeley, CA, USA. The associate editor coordinating the review of this paper and approving it for publication was Y. Sanada.

L. Zheng and L. Cai are with the Department of Electrical and Computer Engineering, University of Victoria, Victoria, BC V8W 2Y2, Canada (e-mail: zhengl@ece.uvic.ca; cai@ece.uvic.ca).

M. Ni and J. Pan are with the Department of Computer Science, University of Victoria, Victoria, BC V8W 2Y2, Canada (e-mail: mmni@uvic.ca; pan@ uvic.ca).

C. Ghosh and K. Doppler are with the Nokia Research Center, Berkeley, CA 94704 USA (e-mail: chittabrata.ghosh@nokia.com; klaus.doppler@ nokia.com).

Color versions of one or more of the figures in this paper are available online at http://ieeexplore.ieee.org.

Digital Object Identifier 10.1109/TWC.2014.2337315

${ }^{1}$ The performance of the GS-DCF in the RAW slot no-crossing case has been studied and evaluated. In this paper, to consider a uniform model for both the RAW slot crossing and no-crossing cases, we have substantially extended the previous model for both RAW slot no-crossing and crossing cases and studied the GS-DCF with different grouping schemes.

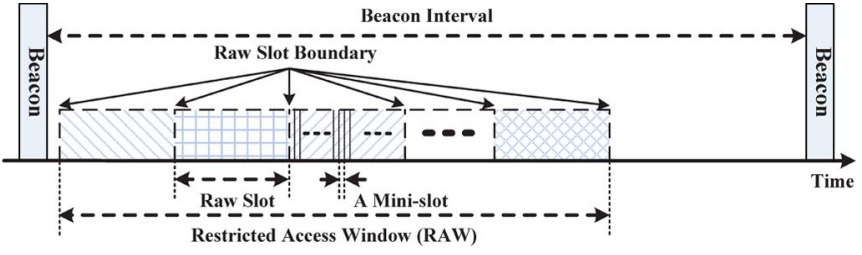

Fig. 1. Structure of the restricted access window (RAW) in IEEE 802.11ah.

of devices use various wireless technologies for two-way communications with a central controller or data collector, which greatly reduces the workload in traditional human-centric data collection processes. Similar scenarios exist in smart gird [2], [3], intelligent transportation systems (ITS), indoor/outdoor surveillance systems, etc. In these scenarios, since the cost of using licensed spectrum to support these new applications is too high, at present, using IEEE $802.11 \mathrm{a} / \mathrm{b} / \mathrm{g} / \mathrm{n}$-like networks is a promising approach. However, the efficiency of the existing contention-based MAC protocols will soon encounter challenges when the network is densely deployed, e.g., an IEEE 802.11ah WLAN is expected to support up to 6,000 stations (STAs) [4]. Moreover, given the large-scale measurement data from several cities, [5] showed that it is common to have tens of access points (APs) deployed in close proximity of each other, which also confirmed the severe contention problem in current and future IEEE 802.11 networks.

To solve the contention problem in a dense network, one strategy is to limit the number of STAs participating in the channel contention at any time by grouping. The idea has been adopted by the IEEE 802.11ah Task Group targeting at the sub- $1 \mathrm{GHz}$ spectrum, specifically $900-928 \mathrm{MHz}$. In the latest draft of the IEEE 802.11ah standard [6], a group-based medium access mechanism is introduced. We term this medium access method as "Group-Synchronized Distributed Coordination Function (GS-DCF)." Using GS-DCF, only the designated stations (STAs) are allowed to access the channel using the prevalent enhanced distributed channel access (EDCA) [7] in a restricted medium access interval, which is termed as restricted access window (RAW), as shown in Fig. 1. Meanwhile, other unassigned groups of STAs are prohibited from medium access within this RAW. One or more RAWs may be allocated within a beacon interval. Besides, a RAW can be further divided into RAW slots, which are further allocated to the different groups of designated STAs.

In fact, the idea of grouping network nodes has been widely used (e.g., the clustering strategy in wireless sensor networks and wireless ad hoc networks [8]-[10]) to increase energy efficiency, decrease management complexity, and optimize 
other network performance metrics. Based on how the groups are organized, the grouping schemes can be categorized into centralized and decentralized ones. Generally speaking, the centralized scheme provides more accurate and fast grouping, but relies on the pre-established network infrastructure and requires extra control signaling to manage groups. In contrast, a decentralized scheme can be more cost-effective in control overhead and more suitable for a dynamic network scenario. To determine a practical choice of the grouping scheme, a thorough performance comparison between these two types of grouping schemes is needed.

In this paper, focusing on modeling the media access performance using GS-DCF and methods of grouping, our main contributions are three-fold. First, we propose an analytical model for the GS-DCF in a saturated network. Second, we analytically study the throughput of GS-DCF using centralized and decentralized grouping schemes when the number of groups in a network is given. In the centralized scheme, STAs are assigned to groups uniformly by AP, and in the decentralized scheme, STAs randomly select the group to join. Third, extensive simulations are performed to validate the proposed models and study the GS-DCF performance in dense networks with different group configurations.

The rest of this paper is organized as follows. We overview the related work in the area of dense 802.11 networks in Section II. The system model is presented in Section III. In Section IV, we propose the analytical model for the GS-DCF under saturated traffic. In Section V, we develop the network performance models when centralized and decentralized grouping schemes are adopted for GS-DCF. Section VI presents the simulation results of model validation and GS-DCF performance evaluation in dense IEEE 802.11 networks with a large number of STAs. Concluding remarks are given in Section VII.

\section{RELATED WORK}

In dense IEEE 802.11 networks, the service received by a STA highly depends on the contention level among co-channel competitors covered by the same or nearby APs. To address the contention problem, many schemes have been proposed in the literature from different perspectives, e.g., using power control [5], [11], rate adaptation [12]-[14], and channel assignment and user association [15]-[18].

The collision problem in IEEE 802.11 networks is essentially the interference problem. With a higher density of STAs and APs, the interference and collisions become severer, which degrade the system performance. As a result, one intuitive solution is to use power control and rate adaptation, which have been demonstrated to yield a substantial improvement in the throughput performance metric. In [5], Akella et al. proposed a power control algorithm called PERF that tunes the transmission power of an AP such that it can support all its clients at the highest transmission rate. In [11], a distributed cross-layer power control algorithm was designed to assign higher transmission power to the cells that are more heavily loaded and tune the transmission power of the physical layer and the carrier sensing parameter of the MAC layer together. In [12], an algorithm to adjust the transmission power and data rate based on the perceived SINR was proposed. In [13], a robust rate adaptation algorithm (RRAA) was proposed to use the short-term packet loss ratio to opportunistically guide its rate adaptation decisions and an adaptive RTS to prevent collision losses from triggering rate reduction. Reference [14] adopted an approach to distinguish the packet losses due to channel fading or collision and infer the collision probability by measuring the contention level, and then to select the transmission rate which maximizes the throughput.

Meanwhile, as IEEE 802.11 provides multiple orthogonal or partially overlapping channels on different frequency bands, how to fully utilize them becomes another important approach to reduce the channel contention in dense networks. In [15], a game-theoretic approach was proposed for the joint channel assignment and user association problem in 802.11 wireless networks, with the objective of balancing the traffic load of different APs. Reference [16] also adopted a game-theoretic approach for a distributed channel allocation. By using channel hopping, [17] proposed a distributed channel assignment algorithm, which can effectively exploit both of the orthogonal and the partially-overlapped channels. A survey on channel assignment schemes for infrastructure-based 802.11 networks can be found in [18].

In summary, to deal with the contention problem in dense IEEE 802.11 networks, the approaches above focus on relieving the impact of channel contention by improving the communication efficiency when there is a winner in the channel contention, however, they cannot limit the channel contentions. Different from these approaches, IEEE 802.11 ah Task Group adopted the grouping strategy for its operation in the sub-1 GHz spectrum. ${ }^{2}$ The basic idea is to divide STAs into groups and let different groups access the channel in a predefined order. Thus, the channel will only be shared by a group of STAs and the channel contentions can be reduced.

An idea similar to the grouping is clustering [8], [9]. In a clustering scheme, according to different criteria, e.g., STAs' mobility, energy-efficiency, or load-balancing, STAs are often organized into clusters based on their geographical locations. Typically, a cluster-head, either fixed or dynamic, will be selected in a cluster to coordinate the communications within the cluster. A comprehensive survey on clustering schemes can be found in [10]. Comparing the clustering and grouping strategies, these two actually attempt to solve the problem on different radio resource dimensions, i.e., grouping exploiting the time dimension and clustering utilizing the space dimension. However, considering the STAs are likely to be close to each other in a dense network, the benefits of clustering may be limited. Besides, location information and explicit messages are required in clustering strategies to maintain the cluster hierarchies, which may consume considerable bandwidth and drain STAs energy quickly.

In the literature, another approach to reduce the channel contention is to use an enlarged contention window [19], [20].

\footnotetext{
${ }^{2}$ Note that IEEE 802.11ah Task Group adopts the grouping as an enhancement over the existing CSMA/CA protocol with EDCA in scenarios with a large number of STAs and is not replacing the existing IEEE 802.11 DCF/EDCF protocol used in IEEE $802.11 \mathrm{a} / \mathrm{b} / \mathrm{g} / \mathrm{n} / \mathrm{ac}$.
} 
In $\mathrm{DCF}$, contention window $(\mathrm{CW})$ is used to reduce the collision probability and it is doubled when a collision happens until a maximum value. As studied in [20], the optimal value of the minimum CW depends on the number of STAs in the network, i.e., $C W_{\text {opt }}=\bar{n} \sqrt{2 T}$, where $\bar{n}$ is the number of active STAs and $T$ is the total packet transmission time including the packet headers, SIFS, ACK and DIFS. However, the limitation of such a method is that it may not be practical in a dense network targeted by IEEE 802.11ah. For example, 6,000 STA will require the optimal minimum CW larger than 12,000 slots, which may introduce unnecessary channel idle time and reduce efficiency. Besides, in dense networks, collisions are more likely to happen due to hidden terminals. In that case, a large $\mathrm{CW}$ cannot effectively reduce collisions, and it may even reduce the channel utilization. In contrast, grouping strategy can be more effective by reducing the channel contention and mitigating the hiddenterminal problem through a proper grouping.

Previously, there have been lots of work on the performance analysis of contention-based channel access in IEEE 802.11 networks. Bianchi firstly proposed a discrete-time Markovchain model to obtain the saturated throughput of the distributed coordination function (DCF) [20]. Following his work, several papers appeared to extend his model to consider different practical issues [21], [22]. Different from Bianchi's approach, a mean value analysis-based approach, which evaluates the average value of system variables, such as STA transmission probability, collision probability and packet service time, without considering the details of the stochastic backoff process, was adopted by [23]-[26]. However, these efforts are for the scenario that all STAs contend the channel simultaneously. To the best of our knowledge, none of them has discussed the impact of the handover between groups and the slot boundary crossing condition, which are introduced in GS-DCF and cause the throughput to deviate substantially from the existing DCF and CSMA rules for wireless channel access [1]. Thus, it is not viable to model the throughput of a given group by treating the slots assigned to other groups as a busy slot and directly applying those previous models. Moreover, how to optimize the MAC configurations for GS-DCF, i.e., the number of groups and RAW slots, the duration for each RAW slot, and the RAW slot allocations, is still an open issue.

\section{System ModeL}

In this paper, we consider a fully-connected IEEE 802.11 network with $N$ STAs accessing the wireless channel within a RAW, i.e., there is no hidden terminal among the $N$ STAs. All packets are delivered from the source to the destination in one hop. The channel time is divided into mini-slots with duration $\delta$. We also assume an ideal channel condition, which does not have communication errors or capture effect, and all packets have the same length.

There are $K$ groups of STAs with group size of $G_{k}(k=$ $\left.1,2, \ldots, K ; \sum_{k=1}^{K} G_{k}=N\right)$. The duration of a RAW and RAW slot is denoted as $T_{R}$ and $T_{s}$, respectively. When there are $K$ groups, $T_{s, k}$ indicates the duration of the RAW slot allocated to the $k$-th group and $T_{R}=\sum_{k=1}^{K} T_{s, k}$. All groups periodically access the channel in their designated RAW slots, and the network is saturated, i.e., all STAs always have packets to transmit.

The channel access process using GS-DCF is similar to that with DCF except that the channel is periodically available to each group. Whenever there is a new packet, a backoff process is invoked. For the $i$-th STA in the $k$-th group, it first senses the channel to be idle for one DIFS with duration $d$ and then chooses a random backoff timer uniformly from $\left[0, C W_{k, i}-\right.$ 1], where $C W_{k, i}$ is the backoff window size. Whenever a collision happens, the backoff window size is doubled from the current size until $C W_{\max }$ is reached. The backoff window is reset to $C W_{\min }$ whenever a packet is acknowledged by the receiver or dropped. When the backoff timer is decreased to zero, the STA obtains a transmission opportunity (TXOP, $\phi$ ), including the transmission time for a packet, an ACK and a SIFS $(\phi=$ DATA + ACK + SIFS $) .{ }^{3}$ Besides, considering the RAW slot to be at least longer than the required time for transmission, i.e., a DIFS, a TXOP, and a mini-slot for backoff counting, it is assumed that $T_{s}>\phi+d+1$. In addition, each packet is allowed to be sent $R_{\max }$ times before it is dropped. Finally, a STA must separate two consecutive transmissions by a random backoff, even if the channel is idle for a DIFS after its previous TXOP [20], [23].

Previously, the analysis work on IEEE 802.11 DCF in the literature, including the Markov-chain-based work [20]-[22] and the mean value analysis-based [23]-[26], adopts the same assumption that the probability for transmitting a packet in an arbitrary slot is the same. Using the same assumption, we study the performance of IEEE 802.11ah GS-DCF, with which the channel is periodically available to a group of STAs and the RAW slot handover of channel between groups plays an important role.

For the notations used in this paper, $\operatorname{Prob}\{A\}$ denotes the probability for event $A$ to happen, $\operatorname{Prob}\{A \mid x\}$ denotes the probability for event $A$ to happen on the condition that a random variable $(R . V)$.$X equal x, P_{X}(x)$ denotes the probability for $X$ equals $x, P_{X \mid Y}(x \mid y)$ denotes the conditional probability for $X$ equals $x$ given another $R . V . Y$ equals $y, E_{X}$ denotes the expectation of $X$, and $E_{X \mid Y}$ denotes the conditional expectation of $X$ given $Y$.

\section{A. Conditional Collision Probability}

For one group of STAs, to obtain the conditional collision probability $p$ given $G=g(g \geq 2)$ STAs, we adopt the approach of mean value analysis, which is similar to that in [26].

Let $E[B]$ and $E[R]$ be the average number of backoff slots and attempts experienced by one packet, respectively. Given that the STA has a packet, the probability to transmit the packet in an idle mini-slot, $\tau$, is

$$
\tau=\frac{E[R]}{E[B]+E[R]} .
$$

\footnotetext{
${ }^{3}$ Note that it is possible for multiple packets to be transmitted within one TXOP in 802.11 networks [7]. In this paper, we focus on studying the GSDCF performance when the network is saturated. Thus, to maintain the fairness among all STAs and for simplicity, it is assumed that only one packet is transmitted in a TXOP and the length of packets is fixed.
} 
For each packet, given the conditional collision probability $p$, the number of transmission attempts follows a truncated geometric distribution with success probability $(1-p)$. Thus, $E[R]$ and $E[B]$ are obtained by

$$
\begin{aligned}
E[R]= & \sum_{r=1}^{R_{\max }-1} r(1-p) p^{r-1}+R_{\max } p^{R_{\max }-1}=\sum_{r=1}^{R_{\max }} p^{r-1}, \\
E[B]= & \frac{1}{2} \sum_{r=1}^{R_{\max }-1} \min \left\{2^{r-1} C W_{\min }, C W_{\max }\right\}(1-p) p^{r-1} \\
& +\frac{1}{2} \min \left\{2^{R_{\max }-1} C W_{\min }, C W_{\max }\right\} p^{R_{\max }-1} \\
= & \frac{1}{2} \sum_{r=1}^{R_{\max }} \min \left\{2^{r-1} C W_{\min }, C W_{\max }\right\} p^{r-1} .
\end{aligned}
$$

Given that a tagged STA transmits in a mini-slot, a collision will happen if any other STA also transmits in the same minislot. Considering the network is saturated and the probability for each STA to transmit is $\tau$, the conditional collision probability is

$$
p=1-(1-\tau)^{g-1} .
$$

Solving (1) and (4) by numerical techniques, we can obtain $p$. As a result, the probability for a packet to be successfully transmitted, $P_{\mathrm{suc} \mid G}$, equals [20]

$$
P_{\mathrm{suc} \mid G}(1 \mid g)=\frac{g \tau(1-\tau)^{g-1}}{1-(1-\tau)^{g}} .
$$

\section{B. Medium Access Within a RAW Slot}

In this paper, we adopt the similar assumption in [1] and [27] that the number of backoff slots a STA has to go through before obtaining a TXOP follows a geometric distribution with the minimum value of one slot. The validation for such an assumption can be found in Section IV-A.

1) Distribution of the Number of Backoff Slots: Let $n_{b}$ denote the number of backoff slots that a STA waits for its TXOP and it follows the geometric distribution,

$$
\operatorname{Prob}\left\{n_{b}=j\right\}=\tau(1-\tau)^{j-1}, \quad(j \geq 1) .
$$

Let $T_{b}$ denote the number of backoff slots between consecutive TXOPs in a RAW slot, $T_{b}$ equals the minimum value of $g$ STAs' backoff counters and it also follows a geometric distribution with the parameter $q^{\prime}=1-(1-\tau)^{g}$, and

$$
P_{T_{b} \mid G}(j \mid g)=q^{\prime}\left(1-q^{\prime}\right)^{j-1}, \quad(j \geq 1, g \geq 2) .
$$

2) Distribution of the Duration for Multiple Transactions: Define a transaction as the sum of a TXOP, DIFS, and the mini-slots used for backoff before the TXOP. A transaction is successful if a packet is successfully received without collision; otherwise, it is failed. Let $T_{b, m}$ denote the number of minislots before the $m$-th TXOP in a RAW slot, and $T_{t, m}$ denote the duration for $m$ transactions. Then,

$$
T_{t, m}=\sum_{m^{\prime}=1}^{m}\left(\phi+d+T_{b, m^{\prime}}\right) .
$$

Note that the sum of $m$ geometric distributed variables, $\sum_{m^{\prime}=1}^{m} T_{b, m^{\prime}}\left(m^{\prime}=1, \ldots, m\right)$, follows a negative binomial distribution with parameters $m$ and $1-q^{\prime}$. Let $\phi^{\prime}=\phi+$ $d$, we obtain the probability for $T_{t, m}$ taking $z$ mini-slots $\left(z \geq m\left(\phi^{\prime}+1\right)\right)$ by (8), that

$$
\begin{aligned}
& P_{T_{t, m} \mid G}(z \mid g)=\operatorname{Prob}\left\{\sum_{m^{\prime}=1}^{m} T_{b, m^{\prime}}=z-m^{\prime} \phi^{\prime} \mid g\right\} \\
& =\left(\begin{array}{c}
z-m \phi^{\prime}-1 \\
z-m \phi^{\prime}-m
\end{array}\right) q^{\prime m}\left(1-q^{\prime}\right)^{z-m \phi^{\prime}-m}, \quad(g \geq 2) .
\end{aligned}
$$

\section{Medium Access Between RAW Slots}

When STAs access the channel within a RAW, one issue that needs to be addressed is the RAW slot handover between two STA groups. According to the current IEEE 802.11ah draft [6], there are two cases in terms of whether an on-going transaction is allowed to cross the RAW slot boundary or not. In the first case (we call it the RAW slot no-crossing case), no transmission is allowed to cross the RAW or RAW slot boundary; in the second case, an on-going transmission is allowed to cross its RAW slot boundary but STAs in the current group should not start a new transmission in the RAW slot allocated to the next STA group (we call it the RAW slot crossing case). Correspondingly, we name the GS-DCF used for the RAW slot no-crossing case and the RAW slot crossing case as NCR GSDCF and CR GS-DCF, respectively.

1) NCR GS-DCF: According to [6], if a STA's on-going transmission may cross the RAW slot boundary, it should hold its transmission and wait for the next available RAW slot. To ensure no transmission crossing the RAW slot, a period of at least $\phi^{\prime}-1$ mini-slots should be set at the end of each RAW slot to separate it from the next one. Besides, to protect the next RAW slot, there is a guard time with duration of $T_{g}$ at the end of each RAW slot in case a transaction may cross the boundary due to synchronization errors or a long propagation delay.

Note that, when multiple packets are sent within one TXOP in real networks, the duration of TXOP can be different. In that case, one approach to set the duration of holding period is to use the maximum allowed TXOP duration; however, it may reduce the channel utilization. Another approach is to adapt the holding period to TXOP required by different STAs. The optimal setting of the holding period is out of the scope of this paper and we leave it as an important future research issue.

As shown in Fig. 2(a), a RAW slot can be divided into two periods, the free access period and the holding period $T_{h}=\phi-$ $1+T_{g}$. Thus, the transmission of a packet can only start during the free access period, and should be completed before the end of the current RAW slot.

During the holding period, while no STA is allowed to start a transaction, there can be multiple options for the operations related to the backoff process: $a$ ) all STAs continue to count down their backoff counters and hold on the transmission to the next available RAW slot if their backoff counters reduce to zero within the holding period; $b$ ) all STAs continue to count down their backoff counters and renew backoff counters with an enlarged contention window by treating the zero backoff 

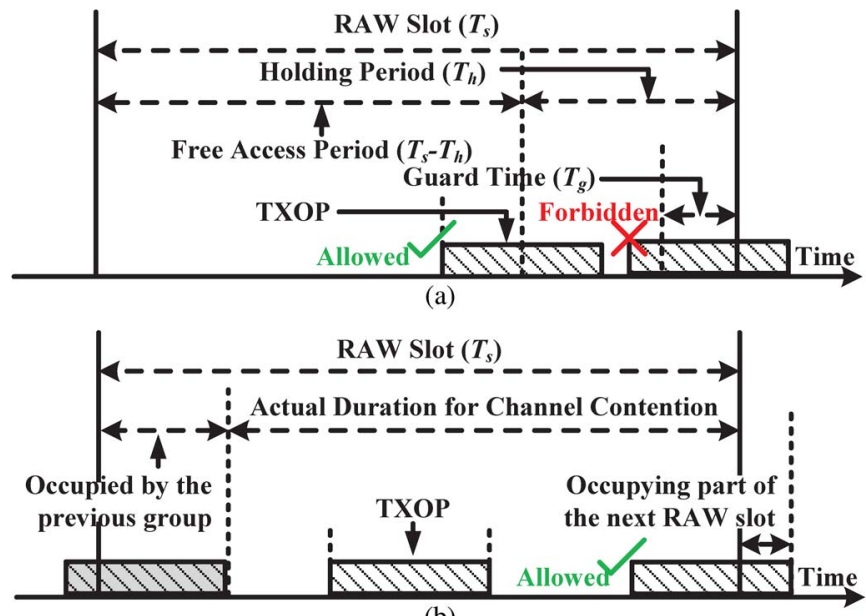

(b)

Fig. 2. Two cases of the media access between RAW slots. (a) RAW slot nocrossing case. (b) RAW slot crossing case.

counters as a virtual collision [26]; c) all STAs continue to count down their backoff counters and renew backoff counters with the same contention window when their backoff counters reduce to zero within the holding period; $d$ ) all STAs freeze their backoff counters during the holding period and resume them in the next available RAW slot.

For the option $a$ ), it is possible for several STAs to count their backoff counters down to zero during the holding time. Thus, the pending transactions are cumulated during the holding period, which may cause severe collisions at the beginning of the next available RAW slot. For the option $b$ ), it is reasonable to dispatch STAs' cumulated pending transactions with more space. However, it inevitably leads to more mini-slots used for backoff. In contrast, options $c$ ) and $d$ ) are more desirable for NCR GS-DCF. Moreover, they are almost equivalent as the backoff counter renewal in option $c$ ) is memoryless. In this paper, we study the NCR GS-DCF with option $d$ ). As a result, the holding period can only be utilized by a transaction if its TXOP starts before the holding period.

2) $C R G S-D C F$ : In this case, a transaction starting at the end of the last RAW slot is allowed to cross the RAW slot boundary. When a group of STAs wake up to access the channel with EDCA, the channel may be occupied by the prior group as shown in Fig. 2(b). As a result, the actual RAW slot duration for one group to contend for the channel in turn may be smaller than the duration of the designated RAW slot. Moreover, comparing with the channel access process using DCF without RAW, there will be channel time wasted for a DIFS if a STA was counting down its backoff counter at the end of its last available RAW slot. Note that on average the channel time available for the transactions of a group is still the same as the duration of the designated RAW slot as their transactions may also occupy part of the next group's RAW slot.

\section{Analytical Models of Saturated GS-DCF}

In this section, we study the throughput of IEEE 802.11 GSDCF under saturated traffic given the group size as $g(g \geq 2)$ in both RAW slot no-crossing and crossing cases.

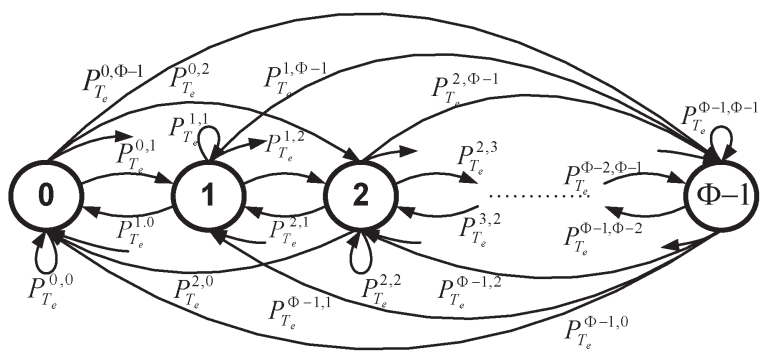

Fig. 3. Markov chain for $T_{e}^{h}$.

\section{A. Actual Duration of a RAW Slot for Channel Contention}

Due to the RAW slot handover between groups, one difference between GS-DCF and DCF is that the channel will not always be available for STAs to contend for TXOP using the former. As a result, in both RAW slot no-crossing and crossing cases, the actual duration of a RAW slot available for channel contention, $T_{s}^{\prime}$, is shorter than the duration of the RAW slot $\left(T_{s}^{\prime} \leq T_{s}\right)$.

1) NCR GS-DCF: In the RAW slot no-crossing case, to avoid a transaction to cross the RAW slot boundary, the holding period $T_{h}$ is unavailable for STAs to contend for channel access. Thus, $T_{s}^{\prime}$ is actually reduced to $T_{s}^{\prime}=T_{s}-T_{h}$.

2) $C R G S-D C F$ : Different from the RAW slot no-crossing case, there is no holding period at the end of a RAW slot in the RAW slot crossing case, however, $T_{s}^{\prime} \leq T_{s}$ in that several minislots at the beginning of the current RAW slot may be occupied by the last transaction initiated in the prior RAW slot. Besides, the difference between $T_{s}^{\prime}$ and $T_{s}$ is an R.V. in the RAW slot crossing case.

Let $T_{e}$ be the number of mini-slots occupied by the last transaction in the previous RAW slot $\left(0 \leq T_{e} \leq \phi-1\right)$, the actual RAW slot duration for channel contention is $T_{s}^{\prime}=T_{s}-T_{e}$.

Assuming that each RAW slot is statistically the same in the long term for a given grouping scheme, $T_{e}$ for different groups are identically distributed, which can be modeled using a Markov chain. As shown in Fig. 3, let the state variable $e$ be the number of mini-slots occupied by the transaction starting in the prior RAW slot $(e=0,1, \ldots, \phi-1), \pi$ be the steady-state probability vector $(\pi=[\pi(0) \pi(1) \cdots \pi(\phi-1)])$, and $P_{T_{e}}$ be the state transition probability matrix. We have

$$
\pi=\pi \cdot P_{T_{e}}
$$

where

$$
P_{T_{e}}=\left[\begin{array}{cccc}
P_{T_{e}}^{0,0} & P_{T_{e}}^{1,0} & \ldots & P_{T_{e}}^{\phi-1,0} \\
P_{T_{e}}^{0,1} & P_{T_{e}}^{1,1} & \ldots & P_{T_{e}}^{\phi-1,1} \\
\cdots & \ldots & \ldots & \cdots \\
P_{T_{e}}^{0, \phi-1} & P_{T_{e}}^{1, \phi-1} & \ldots & P_{T_{e}}^{\phi-1, \phi-1}
\end{array}\right]
$$

and $P_{T_{e}}^{e^{\prime}, e}$ denotes the probability for state $e$ to transit to state $e^{\prime}$. Note that the states' transition probabilities will be affected by the number of STAs and the actual duration of the RAW slot for channel contention will depend on the adopted grouping scheme. We will discuss how to obtain $P_{T_{e}}^{e^{\prime}, e}$ in Section V. 


\section{B. Distribution of the Number of Transactions in a RAW Slot}

Given $T_{s}^{\prime}$ for STAs to contend for TXOPs, the available transmission opportunities within a RAW slot will be limited by $M_{U}\left(T_{s}^{\prime}\right)$ as a function of $T_{s}^{\prime}$. In both cases,

$$
M_{U}\left(T_{s}^{\prime}\right)=\left\lfloor\frac{T_{s}^{\prime}}{\phi^{\prime}+1}\right\rfloor+I_{\left\{T_{s}^{\prime}>\left\lfloor\frac{T_{s}^{\prime}}{\phi^{\prime}+1}\right\rfloor\left(\phi^{\prime}+1\right)+d+1\right.},
$$

where $I_{\{x\}}$ is an indicator function. Here, $I_{\{x\}}=1$ if $x>0$ is true and $I_{\{x\}}=0$, otherwise.

In a RAW slot, let $M$ be the R.V. that indicate the number of transactions within $T_{s}^{\prime}$ with its value of $m$ and $P_{M \mid G}(m \mid g)$ be the probability for there being $m$ transactions. We obtain following results.

a. $M=0$ : if the backoff timers of all STAs are longer than $T_{s}^{\prime}-d$, no STA can send its packet in the current RAW slot. Thus,

$$
\begin{aligned}
& P_{M \mid G}(0 \mid g) \\
& =\operatorname{Prob}\left\{T_{b, 1} \geq T_{s}^{\prime}-d+1\right\}=\sum_{j=T_{s}^{\prime}-d+1}^{C W_{\max }-1} P_{T_{b} \mid G}(j \mid g) .
\end{aligned}
$$

b. $M=1,2, \ldots, M_{U}\left(T_{s}^{\prime}\right)$ : if there are at least $m$ transactions starting during $T_{s}^{\prime}$, it means that the $m$-th transaction starts before the current RAW slot boundary and

$$
\begin{aligned}
& \operatorname{Prob}\{M \geq m\} \\
& \quad=\operatorname{Prob}\left\{T_{t, m-1}+d+T_{b, m}+1 \leq T_{s}^{\prime}\right\} \\
& =\sum_{z=m}^{T_{s}^{\prime}-(m-1) \phi^{\prime}-d-1} \operatorname{Prob}\left\{\sum_{j=1}^{m} T_{b, j}=z \mid g\right\}, \quad(g \geq 2) .
\end{aligned}
$$

Thus, when there are $m$ transactions initiating within $T_{s}^{\prime}$,

$$
P_{M \mid G}(m \mid g)=\left\{\begin{array}{c}
\operatorname{Prob}\{M \geq m\}-\operatorname{Prob}\{M \geq m+1\}, \\
\text { when } 1 \leq M \leq M_{U}\left(T_{s}^{\prime}\right)-1 ; \\
\operatorname{Prob}\left\{M \geq M_{U}\left(T_{s}^{\prime}\right)\right\} \\
\quad \text { when } M=M_{U}\left(T_{s}^{\prime}\right) .
\end{array}\right.
$$

\section{Throughput for a Group of $g(g \geq 2)$ STAs}

With (13)-(15), the expected number of transactions for a group of $g(g \geq 2)$ STAs within a RAW slot, $E_{M \mid G}$, is a function of $g$. Let $E_{M \mid G}^{\mathrm{nc}}$ denote the number in the RAW slot no-crossing case and $E_{M \mid G}^{\mathrm{c}}$ denote the number in the RAW slot crossing case. We have, respectively

$$
\begin{aligned}
& E_{M \mid G}(g) \\
& = \begin{cases}E_{M \mid G}^{\mathrm{nc}}(g) & =\sum_{m=1}^{M_{U}\left(T_{s}-T_{h}\right)} m P_{M \mid G}(m \mid g), \\
E_{M \mid G}^{\mathrm{c}}(g) & =\sum_{e=0}^{\phi-1} \sum_{m=1}^{M_{U}\left(T_{s}-e\right)} m P_{M \mid G}(m \mid g) \cdot \pi(e) .\end{cases}
\end{aligned}
$$

With (4) and (16), we obtain the throughput for a group of $g(g \geq 2)$ STAs (normalized to the channel capacity),

$$
\operatorname{Th}(g)=\frac{L}{T_{R}} E_{M \mid G}(g) \cdot P_{\mathrm{suc} \mid G}(1 \mid g), \quad(g \geq 2),
$$

where $L$ is the transmission time for the payload $(L=$ Payload/DataRate), $P_{\operatorname{suc} \mid G}(1 \mid g)$ is the probability for a transaction to be successful, and $E_{M \mid G}(g)$ denotes the expected number of transactions. $E_{M \mid G}(g)=E_{M \mid G}^{\text {nc }}(g)$ in the RAW slot no-crossing case, and $E_{M \mid G}(g)=E_{M \mid G}^{\mathrm{c}}(g)$ in the RAW slot crossing case.

Note that since $T_{s}^{\prime} \leq T_{s}$ in both RAW slot no-crossing and crossing cases, the channel time available for transactions initiated in a RAW slot is also an R.V. On average, the channel time available for transactions in a RAW slot is less than the duration of the RAW slot in the RAW slot no-crossing case, but same as the duration of the RAW slot in the RAW slot crossing case. Accordingly, the throughput using NCR GS-DCF will be a little lower than that using CR GS-DCF.

Proposition 1: CR GS-DCF can always achieve a throughput higher than or equal to that with NCR GS-DCF.

Proof: Let $f(v)=\sum_{m=1}^{M_{U}\left(T_{s}-v\right)} m P_{M \mid G}(m \mid g)$ be a function of $v$. When $v=T_{h}, f(v)=E_{M \mid G}^{\mathrm{nc}}(g)$; when $v=T_{e}, f(v)$ represents the expected number of transactions in a RAW slot in the RAW slot crossing case given $T_{s}^{\prime}=T_{s}-v$.

Intuitively, $f(v) \geq f(\phi-1)$ if $v \leq \phi-1$ as fewer transactions happen within a smaller $T_{s}^{\prime}$. We have

$$
\begin{aligned}
E_{M \mid G}^{\mathrm{nc}}(g)-E_{M \mid G}^{\mathrm{c}}(g) \leq & f(\phi-1)-\sum_{e=0}^{\phi-d} f\left(T_{e}\right) \pi(e) \\
& <f(\phi-1)-f(\phi-1) \sum_{e=0}^{\phi-d} \pi(e)=0,
\end{aligned}
$$

meaning that on average there are fewer transactions starting in the RAW slot no-crossing case than that in the RAW slot crossing case. According to (17), the expected throughput for a group of STAs in the RAW slot no-crossing case is lower than that in the RAW slot crossing case.

\section{GRouping SCHEMES FOR GS-DCF}

In the previous sections, we study the performance of GSDCF with saturated traffic in a RAW slot given the group size $g$. In this section, we study the network performance of GS-DCF when $N$ STAs are divided into $K$ groups given the RAW size as $T_{R}$ using two grouping schemes, a centralized uniform grouping scheme and a decentralized random grouping scheme. Later on, Section VI-B discusses and compares the impact of different group numbers and grouping schemes on the normalized throughput of GS-DCF in both RAW slot nocrossing and crossing cases.

\section{A. GS-DCF Using the Centralized Uniform Grouping Scheme}

First, we assume that the AP knows the active STAs in the network. Thus, to alleviate the channel contention and to keep the fairness [28] among different groups, one fair strategy is the centralized uniform grouping that the AP assigns the $N$ STAs uniformly to $K$ groups $^{4}(g=N / K)$ and allocates the RAW

\footnotetext{
${ }^{4}$ For notational simplicity, we also assumed that $N$ is divisible by $K$. If not, it is possible to have the group size difference of one STA. Our model can be easily extended to consider this case by modifying (18) as a weighted sum of the throughput for different group sizes.
} 
slots evenly to the $K$ groups $\left(T_{s}=T_{R} / K\right)$. The grouping configuration information can be included in the Beacon Frame and broadcast to all $N$ STAs. For each STA, it can sleep to save energy and only wake up during its designated RAW slots.

When $N$ STAs are uniformly assigned to the $K$ groups, the network throughput $\operatorname{Th}_{\text {uni }}(N, K)$ using GS-DCF with the centralized uniform grouping scheme is

$$
\operatorname{Th}_{\mathrm{uni}}(N, K)=\frac{L K}{T_{R}} E_{M \mid G}(g) \cdot P_{\mathrm{suc} \mid G}(1 \mid g) .
$$

For both of the RAW slot no-crossing and crossing cases, $P_{\text {suc } \mid G}(1 \mid g)$ can be computed by (5).

Note that when the group number $(K)$ is the same as the number of STAs $(N)$, there will be only one STA in a group $(g=1)$. In that case, transmissions are always successful as there is no channel contention and the backoff counted between transmissions follows a uniform distribution between 0 and $\left(C W_{\min }-1\right)$ instead of the geometric distribution as what we used in Section IV when $g \geq 2$. Consequently, to obtain $E_{M \mid G}(g)$, (9) has to be modified by considering $\sum_{m^{\prime}=1}^{m} T_{b, m^{\prime}}$ as the sum of $m$ uniformly distributed random variable(s).

In addition, the steady-state distribution of $\pi$ is required to obtain $E_{M \mid G}(g)$ in the RAW slot crossing case. While the channel contention in each RAW slot is statistically the same for the centralized grouping scheme, the state transition matrix can be obtained as follows.

$$
P_{T_{e}}^{e^{\prime}, e}= \begin{cases}1-\sum_{e^{\prime}=1}^{\phi-1} P_{T_{e}}^{e^{\prime}, e}, & e^{\prime}=0 ; \\ \sum_{m=1}^{\left\lfloor\frac{T_{s}-T_{e}+T_{e^{\prime}}}{\phi^{\prime}+1}\right\rfloor} P_{T_{t, m} \mid G}\left(T_{s}-T_{e}+T_{e^{\prime}} \mid g\right), & e^{\prime}>0 .\end{cases}
$$

By (10), (11) and (19), we obtain $\pi$.

Finally, using (10)-(16) and (19), we compute $E_{M \mid G}^{\mathrm{nc}}(g)$ and $E_{M \mid G}^{\mathrm{c}}(g)$, respectively, and then derive the throughput of $N$ STAs using GS-DCF with the centralized uniform grouping scheme using (18).

\section{B. GS-DCF Using the Decentralized Random Grouping Scheme}

While the centralized uniform grouping scheme can guarantee the load balance among different groups, it relies on the assistance of the AP for the channel coordination, which is not consistent with the essential principle behind the contentionbased channel access. Besides, if the number of STAs in a network varies with time, the AP has to regroup STAs frequently, which may introduce a high control overhead. Thus, comparing with the centralized uniform grouping scheme, a grouping scheme allowing STAs to make grouping decisions by themselves may be more desirable in terms of the implementation complexity and control overhead.

We call such a scheme the decentralized random grouping scheme, in which a STA randomly chooses one of the $K$ RAW slots (with the size of $T_{R} / K$ ) with probability $1 / K$ at the beginning of a RAW. Unlike the centralized uniform grouping scheme, only $K$ is decided by the AP and broadcasted to STAs in the beacon message.
TABLE I

PARAmeters USED in Simulation (I) [6]

\begin{tabular}{r|cc}
\hline Parameter & aSlotTime & SIFS \\
\hline Value & $52 \mu \mathrm{s}$ & $160 \mu \mathrm{s}$ \\
\hline
\end{tabular}

TABLE II

PARAMETERS USED IN Simulation (II) [7]

\begin{tabular}{r|ccc}
\hline Parameter & MacHeader & ACK & $T_{\text {PLCP }}$ \\
\hline Value & 34 bytes & 14 bytes & $20 \mu \mathrm{s}$ \\
\hline \hline Parameter & $C W_{\min }$ & $C W_{\max }$ & $R_{\max }$ \\
\hline Value & 16 & 1,024 & 7 \\
\hline
\end{tabular}

With such a grouping scheme, the group size given $N$ STAs in the network becomes an R.V., which follows a binomial distribution with parameters $N$ and $1 / K$. For one group, there are $g$ STAs with probability $P_{G}(g)(g=0,1,2, \ldots, N)$ and we have

$$
P_{G}(g)=\left(\begin{array}{c}
N \\
g
\end{array}\right) \frac{(K-1)^{N-g}}{K^{N}} .
$$

Note that it is possible for a group to be empty when STAs randomly select their groups in a distributed way, which reduces the throughput depending on the numbers of STAs and groups.

Thereby, the throughput achieved by $N$ STAs using GS-DCF with the decentralized random grouping scheme is ${ }^{5}$

$$
\mathrm{Th}_{\mathrm{rnd}}(N, K)=\frac{L K}{T_{R}} \sum_{g=1}^{N} E_{M \mid G}(g) \cdot P_{\mathrm{suc} \mid G}(1 \mid g) \cdot P_{G}(g) .
$$

To obtain $E_{M \mid G}(g)$ in (21), we use the same method as that for the centralized uniform grouping scheme. Note that it does not give the exact solution. In practice, as STAs may join groups randomly using the decentralized grouping sheme, the channel contention experienced by a tagged STA is different from that when the STA continuously competes with a fixed group of STAs. One example is the case when there is only one STA in a group. With a fixed group of STAs contend for the wireless channel, the transaction will always be successful and the STA's contention window is always $C W_{\min }$ if $T_{s}^{\prime}>C W_{\min }+d+\phi$. However, when different STAs join a group at different time, no transaction may happen if $\left(T_{s}^{\prime}-d-\phi\right)$ is larger than $C W_{\min }$ but less than a STA's current backoff counter due to continuous packet collisions in the previous RAW slots. In Section VI-A, the accuracy of such an approximation is evaluated.

\section{Performance Evaluation And AnAlysis}

Simulations are carried out with the PHY and MAC layer parameters in the IEEE 802.11ah draft [6] and IEEE 802.11 standard [7] as listed in Tables I and II, respectively. In the simulation, due to the traffic pattern of 802.11ah usage model, e.g., smart grid, we set the payload size to a relatively small value, which is 64 bytes and the data rate is $1 \mathrm{Mbps}^{6}$ The TXOP

\footnotetext{
${ }^{5}$ Note that, although the group sizes between $K$ RAW slots are correlated as their sum is fixed at $N$, such correlation will not affect the average throughput.

${ }^{6}$ According to IEEE 802.11ah draft, GS-DCF will be used in the scenario where an AP will support a large number of STAs scattering in an large area, e.g., smart grid or sensor networks with coverage of $1 \mathrm{~km}$. Thus, $1 \mathrm{Mbps}$ was used in simulation to ensure the coverage.
} 


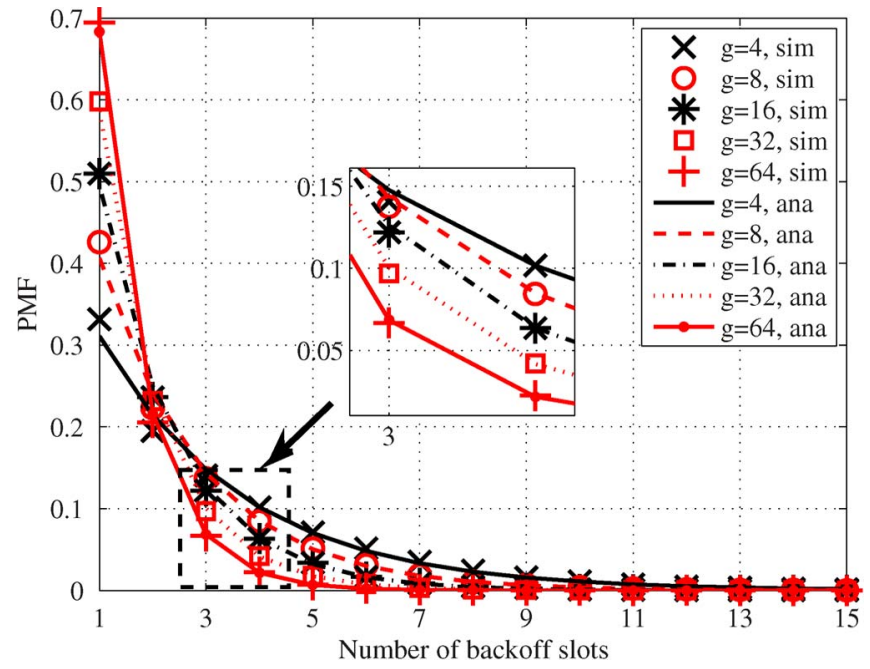

Fig. 4. PMF of the number of backoff slots between transactions.

is $1.1 \mathrm{~ms}$, which can accommodate one packet transmission, including the data transmission time, a SIFS and the ACK transmission time. The duration of RAW is set as $500 \mathrm{~ms},{ }^{7}$ which is sufficient to accommodate at least one transaction when the number of groups $(K)$ is up to $256(500 \mathrm{~ms} / 256 \approx$ $1.953 \mathrm{~ms}>$ aSlotTime + DIFS $+\phi=1.404 \mathrm{~ms})$.

$$
\begin{aligned}
\phi & =T_{\mathrm{DATA}}+\mathrm{SIFS}+T_{\mathrm{ACK}}=1.1 \mathrm{~ms}, \\
T_{\mathrm{DATA}} & =T_{\mathrm{PLCP}}+\frac{\text { Payload }+ \text { MacHeader }}{\text { DataRate }}=0.804 \mathrm{~ms}, \\
T_{\mathrm{ACK}} & =T_{\mathrm{PLCP}}+\frac{\text { ACK }}{\text { DataRate }}=0.132 \mathrm{~ms},
\end{aligned}
$$

where $T_{\mathrm{DATA}}$ and $T_{\mathrm{ACK}}$ are the transmission time of a data frame and an immediate-acknowledgement frame, respectively. For the network setting, we assume that there are $N$ STAs in the WLAN. In the following, "UNI" denotes the GS-DCF using the centralized uniform grouping scheme and "RND" indicates that the decentralized random grouping scheme is adopted for GS-DCF.

\section{A. Model Validation}

In this section, we first validate the assumption that the number of backoff slots between transactions has a geometric distribution, and then validate the throughput models for GSDCF using the centralized uniform grouping scheme (18) and the decentralized random grouping scheme (21).

1) The Distribution of the Number of Backoff Slots Between Transactions: Assuming there are 64 groups with $g \in$ $\{4,8,16,32,64\}$ STAs in each group, we compare the probability mass function (PMF) of the number of backoff slots between transactions in simulations and the one estimated by our model in Section III-B. As Fig. 4 shows, the two results match with each other quite well.

\footnotetext{
${ }^{7}$ The duration of RAW depends on the duration of TXOP and the maximum number of groups. For delay sensitive applications, the duration of RAW may be set with a small value. Due to the space limit, we omit the performance evaluation with a small RAW duration.
}

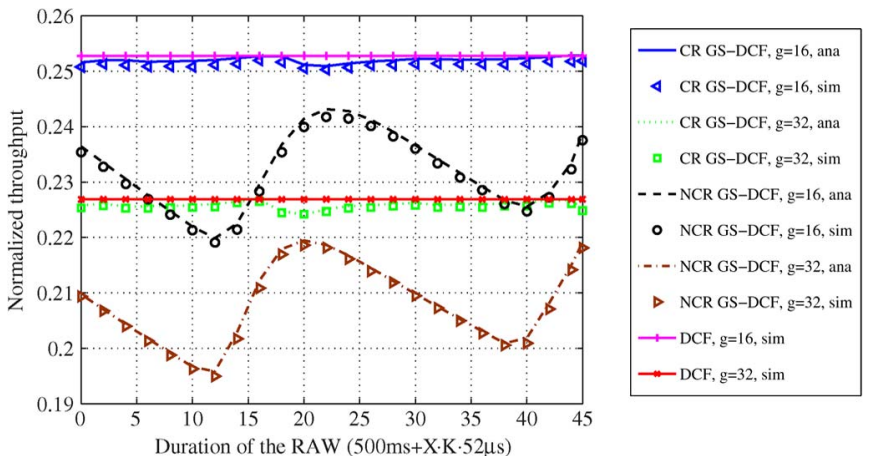

(a)

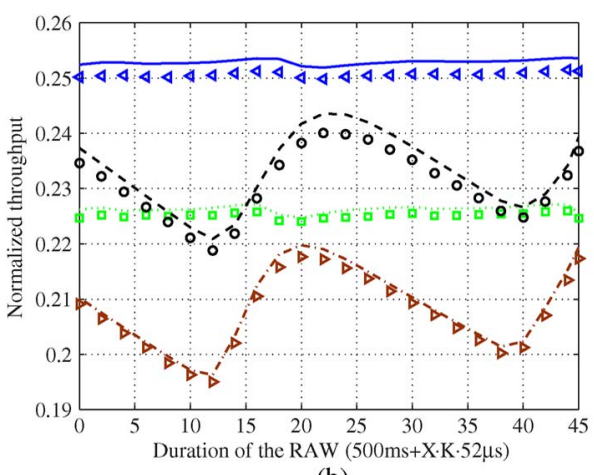

(b)

Fig. 5. Normalized throughput. (a) UNI GS-DCF. (b) RND GS-DCF.

2) Normalized Throughput Using GS-DCF: To validate the proposed model, comparisons are made between the simulation and the analytical results focusing on the normalized throughput for $N$ STAs in the network.

In the simulations, there are $N \in\{1024,2048\}$ STAs uniformly or randomly accessing the media in 64 groups $(g \in$ $\{16,32\})$ with the RAW varying from $500 \mathrm{~ms}$ to $650 \mathrm{~ms}$, i.e., the duration of a RAW slot is increased by aSlotTime per step. Fig. 5 illustrates the average of the normalized throughput using UNI GS-DCF and RND GS-DCF.

First, the results computed by the proposed analytical models match with the simulation quite well with the gap less than $3 \%$. Second, it can be observed that the normalized throughput increases in a fluctuating way as the duration of the RAW slot increases. The reason is that a varying number of mini-slots can be wasted due to the RAW slot handover between groups.

In NCR GS-DCF, mini-slots in the holding period may not be utilized when all STAs hold on their backoff counters. In CR GS-DCF, although there is no holding period, channel utilization is reduced as a transaction may have to wait more than one DIFS, which happens when a group of STAs use the last RAW slot for backoff counting and have to wait an extra DIFS at the beginning of their next RAW slot.

In both cases, due to the limited channel access interval $\left(T_{s}^{\prime}\right)$ and the fact that the probability of the geometrically distributed $T_{b}$ 's (7) decays quickly as $T_{b}$ increases, the number of wasted mini-slots using GS-DCF, which reduces the throughput, varies w.r.t. the duration of a RAW slot. To demonstrate such a phenomena, Fig. 6 illustrates the relationship between the ratio of wasted mini-slots and the corresponding normalized throughput using the centralized uniform grouping scheme for both RAW 


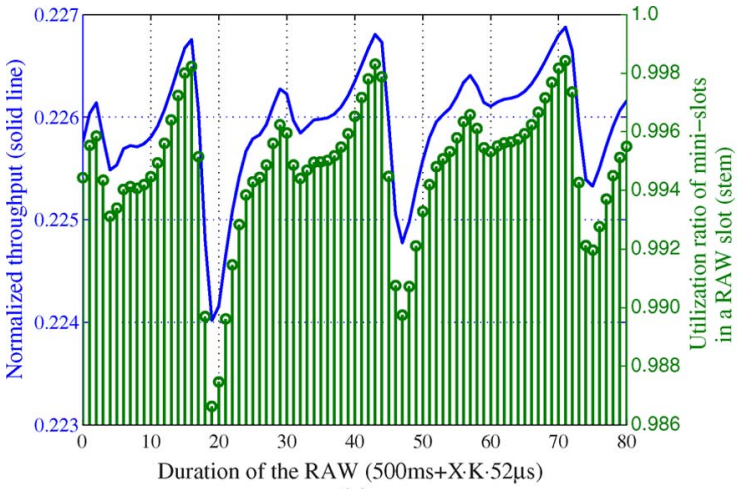

(a)

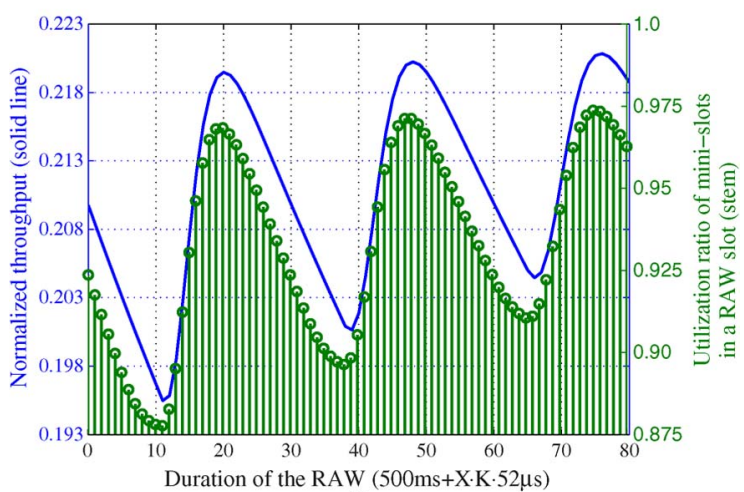

(b)

Fig. 6. Ratio of wasted mini-slots and corresponding normalized throughput using the uniform grouping scheme $(g=16, K=64)$. (a) CR GS-DCF. (b) NCR GS-DCF.

slot crossing and no-crossing cases with 64 groups and 16 STAs per groups.

Note that the number of wasted mini-slots in a RAW slot is bounded by $T_{h}$ in NCR GS-DCF and $d$, the duration of a DIFS, in CR GS-DCF. Thus, the throughput fluctuations are also bounded. In addition, CR GS-DCF not only achieves a higher throughput than NCR GS-DCF, as proved in Section IV-B, the absolute amplitude of the throughput fluctuation in CR GS-DCF is also smaller than that in NCR GS-DCF as shown in Fig. 5.

In Fig. 5(a), it also shows the normalized throughput of $g \in\{16,32\}$ STAs using DCF without considering the RAW slot handover. Note that given $N$ STAs in total, GS-DCF is anticipated to outperform DCF by reducing the channel contention (demonstrated in Section VI-B); however, the normalized throughput using GS-DCF is less than that using DCF with $g$ STAs. If the duration of the RAW slot increases, the negative impact of wasted mini-slots on the normalized throughput diminishes. When the RAW slot is sufficiently large, the normalized throughput obtained is close to what is achievable using DCF.

Fig. 7 shows the normalized throughput of GS-DCF when signal fading is considered. In the simulation, $N \in$ $\{1248,2048\}$ STAs uniformly distribute around the AP within $1 \mathrm{~km}$. The signal transmitting power is $20 \mathrm{dBm}$, and Rayleigh fading is considered with the pathloss and shadowing computed based on the pathloss model for TGah outdoor scenarios [29]. A packet transmission is considered to be successful if the receiv-

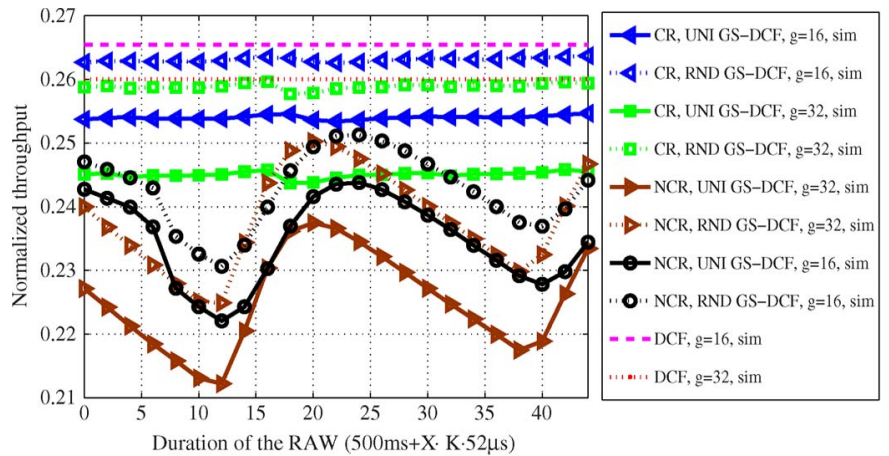

Fig. 7. Normalized throughput in a real network (Pathloss $(\mathrm{dB})=37.6+8$. $\log$ (distance $(m))$ and shadowing with standard deviation of $8 \mathrm{~dB}$ [29]).

ing signal-to-interference-and-noise ratio (SINR) is equal to or higher than $10 \mathrm{~dB}$ depending on the coding and modulation scheme.

Comparing to Fig. 5, the results in Fig. 7 show similar trends when the RAW duration increases. However, due to the signal capture effect, i.e., a collided packet may still be successfully received despite the presence of interference from other (weaker) transmissions [30], the normalized throughputs are slightly higher than those in Fig. 5. Moreover, the throughput gain from the signal capture effect is larger when a larger group size and/or random grouping is used. This is because these two factors both increase the diversity of the signal strength of packets collided together, which allows more packets to be captured among collisions. To quantify the impact of the signal capture effect, the proposed GS-DCF throughput models can be extended by integrating the probability for a capture to happen in (4). For a packet being captured in a collision, the probability can be obtained by computing the probability density function of the largest SINR among all packets by considering the channel and STAs' spatial distribution models [30], [31]. A complete analytical model fully considering the signal capture effect remains a further research issue.

\section{B. GS-DCF in a Dense Network}

Applying the proposed analytical model, we investigate the GS-DCF performance in a dense network using different grouping schemes. In the simulation, $N \in\{256,512,1024,2048\}$ STAs are divided into $K(8,16,32,64,128,256)$ groups.

Fig. 8 shows the normalized throughput when STAs access the wireless media using GS-DCF with different $K \mathrm{~s}$ and the normalized throughput using DCF with $N \in\{256,512\}$ STAs contending for the wireless channel.

1) Comparison Between GS-DCF and DCF: As shown in Fig. 8, by reducing the channel contention, the GS-DCF outperforms DCF prominently in terms of the normalized throughput. There are $210 \%$ and $770 \%$ throughput gains using UNI CR GSDCF in a network with 256 STAs in 128 groups and 512 STAs in 256 groups, respectively.

2) Comparison Among Different Group Configurations for $G S$-DCF: Also, it can be found in Fig. 8 that there is a $K$, which maximizes the throughput using GS-DCF, e.g., $K=128$ for $N=256$ using UNI CR GS-DCF and $N=512$ using RND 


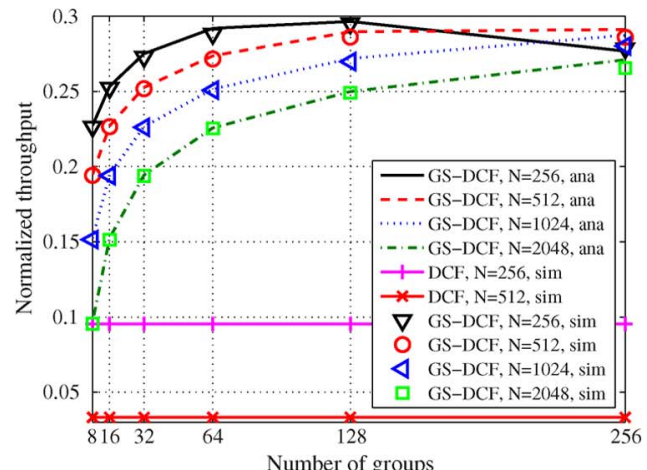

(a)

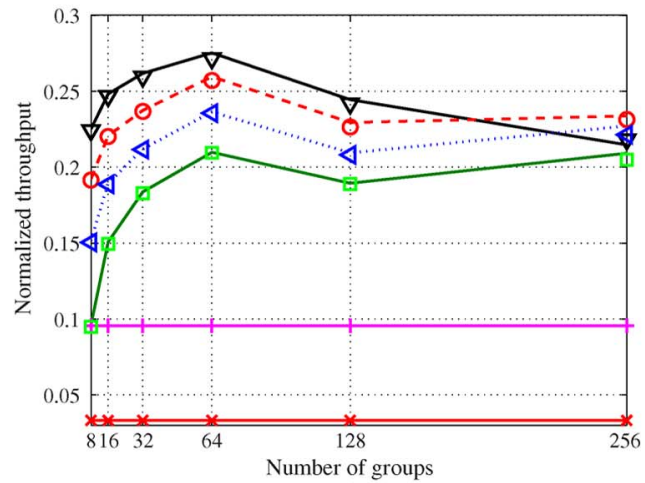

(b)

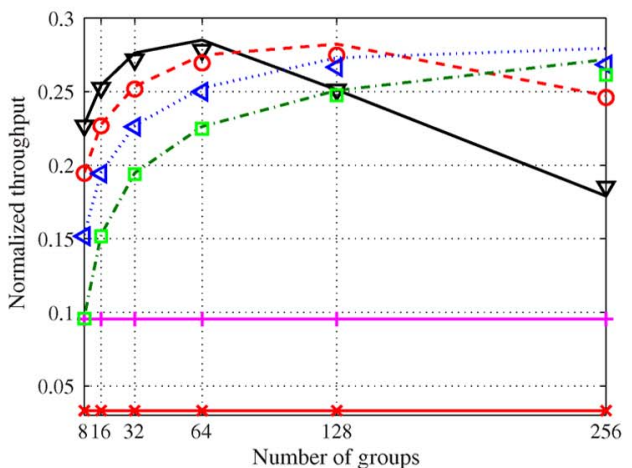

(c)

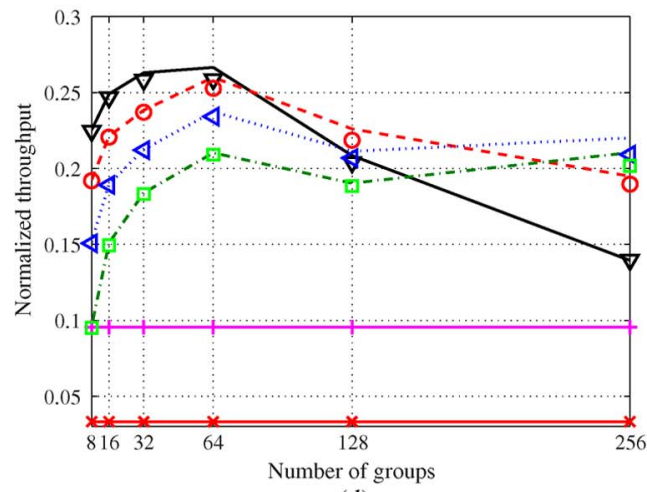

(d)

Fig. 8. Normalized throughput with different numbers of groups. (a) UNI CR GS-DCF. (b) UNI NCR GS-DCF. (c) RND CR GS-DCF. (d) RND NCR GS-DCF.

CR GS-DCF; $K=64$ for $N=256$ using RND CR GS-DCF and all four dense networks using NCR GS-DCF.

When $K$ is relatively small (8-64 groups), the throughput of GS-DCF increases with $K$. This is attributed to the re- duced channel contention. Meanwhile, it is also found that the throughput decreases when $K$ is too large (128, 256 groups). For CR GS-DCF, the throughput in Fig. 8(a) drops when there is only one STA per group using UNI CR GS-DCF $(N=256)$. Two factors cause such a phenomena. First, when the packet transmission time is short $(804 \mu \mathrm{s})$, the duration for the backoff process has a remarkable impact on the throughput. Second, a STA still has to access the channel by following EDCA when it is the only STA in the RAW slot, which makes it possible that the STA spends more mini-slots for the backoff process, e.g., a half of the minimum contention window on average $(416 \mu \mathrm{s})$, than that when there are multiple STAs competing with each other. Note that such a phenomena may not exist when the packet payload size is sufficiently large, e.g., 2,312 bytes (the maximum MAC service data unit, MSDU [7]). Thus, the impact of the backoff duration becomes relatively small and the throughput for one STA per group can be larger than those of the other cases with channel contention. In Fig. 8(c), with RND CR GS-DCF, the throughput drops more with a larger $K$ than that using UNI CR GS-DCF, as shown in the case when $N=256$ and $K=128$. The reason is that the probability for a group to be empty becomes high when $K$ increases. Thus, when $K$ is close to or larger than $N$, some RAW slots with no STA are wasted.

Note that when the group number increases, the throughput variation using NCR GS-DCF can be more complicated than that using CR GS-DCF. For NCR GS-DCF, besides the two factors discussed above, when the duration of RAW slots decreases, the throughput fluctuation due to the wasted minislots becomes more remarkable. For example, it is observed in Fig. 8(b) and (d) that the throughput using NCR GS-DCF with 256 groups, e.g., $N \in\{512,1024,2048\}$ STAs using the centralized uniform grouping scheme and $N \in\{1024,2048\}$ STAs using the decentralized random grouping scheme, is higher than those using 128 groups since the collisions are reduced with more groups.

3) Comparison Between UNI GS-DCF and RND GS-DCF: A decentralized grouping scheme can be more desirable in a practical system to avoid the control overheads introduced using the centralized grouping scheme. The impact of using the decentralized grouping scheme on the normalized throughput is evaluated by simulations and presented in Figs. 9 and 10.

Defining the difference ratio of the normalized throughput as $\left(T h_{\text {uni }}-\mathrm{Th}_{\mathrm{rnd}}\right) / \mathrm{Th}$ uni, Fig. 9 compares RND GS-DCF with UNI GS-DCF using the same $K$ under different network densities. It can be found that the performance of RND GSDCF is quite close to that of UNI GS-DCF when $K$ is small. However, because the possibility to have empty groups using RND GS-DCF increases when $K$ becomes larger, the gap between the two grouping schemes increases.

In Fig. 10, we illustrate the normalized throughput loss by comparing the RND GS-DCF throughput with the maximum throughput obtained using UNI GS-DCF, i.e., $T h_{\text {rnd }} /$ $\max \left\{\mathrm{Th}_{\mathrm{uni}}\right\}$. As shown in the figure, RND GS-DCF can be implemented with less than $6 \%$ throughput loss with a proper choice of $K$.

Note that the value of the throughput loss and the optimal number of groups to implement RND GS-DCF with the 


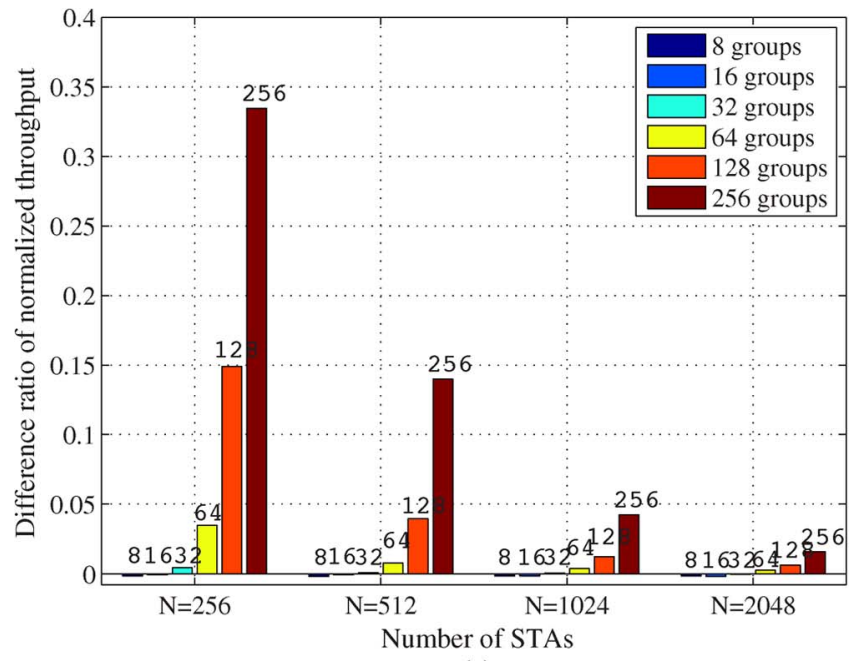

(a)

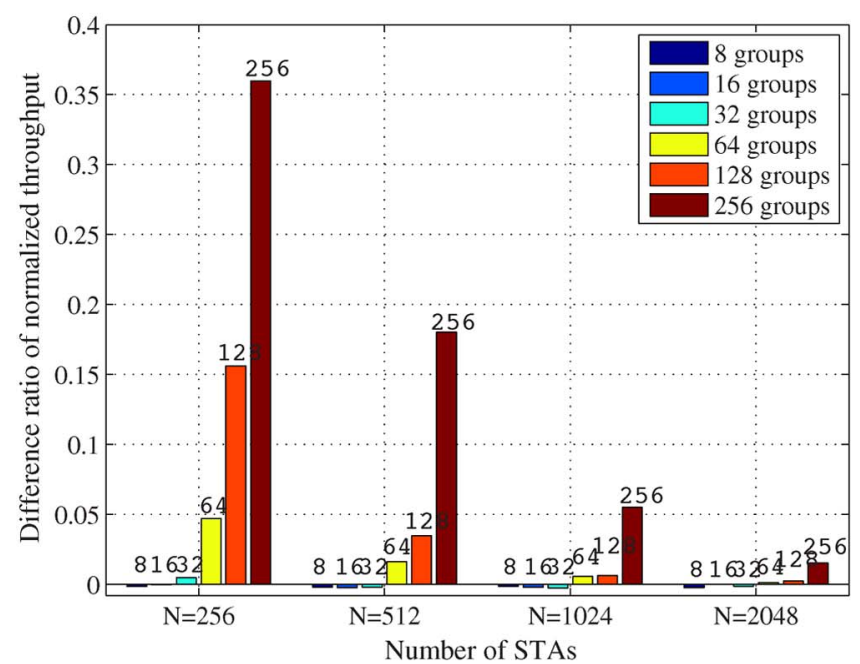

(b)

Fig. 9. Normalized throughput difference ratio. (The numbers above the bar stand for the number of groups.) (a) CR GS-DCF. (b) NCR GS-DCF.

minimum throughput loss are also affected by other factors, e.g., the payload size, the data rate, packet overhead, and MAC parameters (aSlotTime, SIFS, etc). The analytical framework presented in this work can be applied to optimize the group size given the system parameters. Further research efforts are needed for a system with varying payload size and data rate.

\section{CONCLUSION}

In this paper, we have proposed a unified analytical framework for IEEE 802.11ah GS-DCF in both the RAW slot crossing and no-crossing cases. An accurate model for the saturated throughput has been presented, and two grouping schemes, the centralized uniform grouping and the decentralized random grouping, have been discussed and compared. It has been demonstrated that GS-DCF is promising in significantly improving the throughput in dense networks by effectively alleviating the channel contention. Besides, it has been observed that the RAW slot handover in GS-DCF can cause the throughput to fluctuate, and such impact is more prominent in the RAW slot no-crossing case than the RAW slot crossing

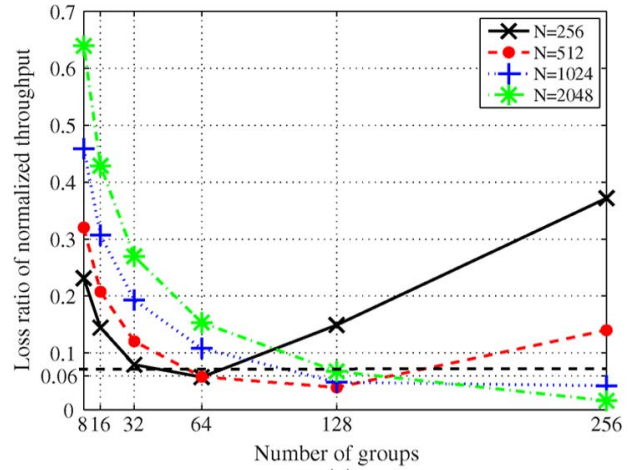

(a)

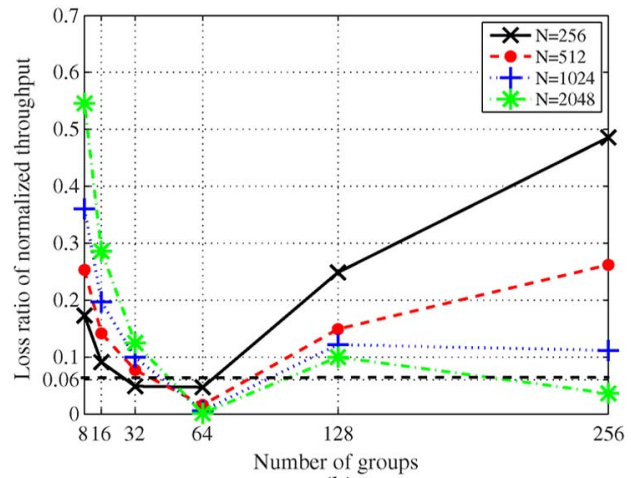

(b)

Fig. 10. Throughput loss using RND GS-DCF comparing with UNI GS-DCF. (a) CR GS-DCF. (b) NCR GS-DCF.

case. In addition, it has also been demonstrated that the decentralized grouping scheme can achieve a similar throughput to that of the centralized grouping scheme in dense networks, which is important to support the distributed implementation of GS-DCF.

There are several issues beckoning for further research. The first is to investigate the performance of GS-DCF in more heterogeneous and dynamic communications scenarios. In this paper, we have focused on the modeling and assumed homogeneous payload size, data rate, and saturated traffic. In practice, both the packet length and data rate can be heterogeneous, the traffic can be unsaturated, and the hidden terminal problems will also affect the system performance. Second, a careful design of the MAC configurations is necessary for GS-DCF. As the throughput using GS-DCF fluctuates with the changes in the duration of RAW slots, it may not be a wise choice to always allocate RAW slots of the same duration for different groups. Besides, the duration of a RAW may be limited considering applications' stringent requirement on communication latency, which leads to short RAW slots and more significant negative impact of the holding period in NCR GS-DCF and the potential extra DIFS in CR GS-DCF on the throughput. Moreover, when the traffic is heterogeneous, the durations of TXOPs for different STAs can be different. As a result, a fixed holding period in the no-crossing case may not be efficient, which needs to be optimized by adaptively setting the holding period for different STAs. To jointly optimize the number of groups and the duration of RAW and RAW slots for GS-DCF, we believe the analytical model developed in this paper can be helpful. Third, as GS-DCF improves the DCF performance in 
IEEE 802.11ah dense network by restricting STAs's channel access through grouping, it can be a good complement to the existing solutions designed for IEEE 802.11 DCF. However, how to effectively combine them in an optimal way to further improve the performance requires more efforts in the future.

\section{REFERENCES}

[1] L. Zheng, L. Cai, J. Pan, and M. Ni, "Performance analysis of grouping strategy for dense IEEE 802.11 networks," in Proc. IEEE GLOBECOM, Dec. 2013, pp. 1-6.

[2] L. Zheng and L. Cai, "A distributed demand response control strategy using Lyapunov optimization," IEEE Trans. Smart Grid, vol. 5, no. 4, pp. 2075-2083, Jul. 2014.

[3] K. Zhou, L. Cai, and J. Pan, "Optimal combined heat and power system scheduling in smart grid," in Proc. IEEE INFOCOM, Apr. 2014, pp. 2831-2839.

[4] IEEE 802.11ah Task Group, TGah Functional Requirements and Evaluation Methodology Rev. 5. [Online]. Available: http://www.ieee802.org/ 11/Reports/tgah update.htm

[5] A. Akella, G. Judd, S. Seshan, and P. Steenkiste, "Self-management in chaotic wireless deployments," in Proc. ACM MOBICOM, Aug./Sep. 2005, pp. 185-199.

[6] IEEE 802.11ah Task Group, 11/1137r14 Specification Framework for TGah. [Online]. Available: http://www.ieee802.org/11/Reports/ tgah_update.htm

[7] Wireless LAN Medium Access Control (MAC) and Physical Layer (PHY) Specifications Amendment 8: IEEE 802.11 Wireless Network Management, IEEE Std. 802.11v-2011, Sep. 2011.

[8] C. Lin and M. Gerla, "Adaptive clustering for mobile wireless networks," IEEE J. Sel. Areas Commun., vol. 15, no. 7, pp. 1265-1275, Sep. 1997.

[9] T.-C. Hou and T.-J. Tsai, "A access-based clustering protocol for multihop wireless Ad Hoc networks," IEEE J. Sel. Areas Commun., vol. 19, no. 7, pp. 1201-1210, Jul. 2001

[10] J. Yu and P. H. J. Chong, "A survey of clustering schemes for mobile ad hoc networks," IEEE Commun. Surveys Tuts., vol. 7, no. 1, pp. 32-48, 2005.

[11] V. Mhatre, K. Papagiannaki, and F. Baccelli, "Interference mitigation through power control in high density 802.11 WLANs," in Proc. IEEE INFOCOM, May 2007, pp. 535-543.

[12] J. Kim, S. Kim, S. Choi, and D. Qiao, "CARA: Collision-aware rate adaptation for IEEE 802.11 WLANs," in Proc. IEEE INFOCOM, Apr. 2006, pp. 1-11.

[13] S. H. Y. Wong, H. Yang, S. Lu, and V. Bharghavan, "Robust rate adaptation for 802.11 wireless networks," in Proc. ACM MOBICOM, Sep. 2006, pp. $146-157$.

[14] K. V. Cardoso and J. F. Rezende, "Increasing throughput in dense 802.11 networks by automatic rate adaptation improvement," Wireless Netw., vol. 18, no. 1, pp. 95-112, Jan. 2012.

[15] W. Xu, C. Hua, and A. Huang, "Channel assignment and user association game in dense 802.11 wireless networks," in Proc. IEEE ICC, Jun. 2011, pp. 1-5.

[16] J. Chen et al., "Game theoretical approach for channel allocation in wireless sensor and actuator networks," IEEE Trans. Autom. Control, vol. 56, no. 10 , pp. $2332-2344$, Oct. 2011

[17] A. Mishra, V. Shrivastava, D. Agrawal, S. Banerjee, and S. Ganguly, "Distributed channel management in uncoordinated wireless environments," in Proc. ACM MOBICOM, Sep. 2006, pp. 170-181.

[18] S. Chieochan, E. Hossain, and J. Diamond, "Channel assignment schemes for infrastructure-based 802.11 WLANs: A survey," IEEE Commun. Surveys Tuts., vol. 12, no. 1, pp. 124-136, 2010.

[19] G. Bianchi, L. Fratta, and M. Oliveri, "Performance evaluation and enhancement of the CSMA/CA MAC protocol for 802.11 wireless LANs," in Proc. PIMRC, Oct. 1996, vol. 2, pp. 392-396.

[20] G. Bianchi, "Performance analysis of the IEEE 802.11 distributed coordination function," IEEE J. Sel. Areas Commun., vol. 18, no. 3, pp. 535-547, Mar. 2000.

[21] J. Robinson and T. Randhawa, "Saturation throughput analysis of IEEE 802.11e enhanced distributed coordination function," IEEE J. Sel. Areas Commun., vol. 22, no. 5, pp. 917-928, Jun. 2004.

[22] C. Hu, H. Kim, J. Hou, D. Chi, and S. Nandagopalan, "Provisioning quality controlled medium access in ultra wide band-operated WPANs," in Proc. IEEE INFOCOM, 2006, pp. 1-11.

[23] Y. C. Tay and K. C. Chua, "A capacity analysis for the IEEE 802.11 MAC protocol," Wireless Netw., vol. 7, no. 2, pp. 159-171, Mar. 2001.
[24] L. Cai, X. Shen, J. W. Mark, L. Cai, and Y. Xiao, "Voice capacity analysis of WLAN with unbalanced traffic," IEEE Trans. Veh. Technol., vol. 55, no. 3, pp. 752-761, May 2006.

[25] R. Zhang, L. Cai, and J. Pan, "Performance analysis of reservation and contention-based hybrid MAC for wireless networks," in Proc. IEEE ICC, 2010, pp. 1-5.

[26] R. Zhang, R. Ruby, J. Pan, L. Cai, and X. Shen, "A hybrid reservation/contention-based MAC for video streaming over wireless networks," IEEE J. Sel. Areas Commun., vol. 28, no. 3, pp. 389-398, Apr. 2010.

[27] A. Abdrabou and W. Zhuang, "Service time approximation in IEEE 802.11 single-hop ad-hoc networks," in Proc. IEEE INFOCOM, May 2007, pp. 2346-2350.

[28] K. Kashibuchi, A. Jamalipour, and N. Kato, "Channel occupancy time based TCP rate control for improving fairness in IEEE $802.11 \mathrm{dcf}, "$ IEEE Trans. Veh. Technol., vol. 59, no. 6, pp. 2974-2985, Jul. 2010.

[29] IEEE 802.11ah Task Group, TGah Channel Model. [Online]. Available: https://mentor.ieee.org/802.11/dcn/11/11-11-0968-01-00ah-channelmodel-text.docx

[30] J. H. Kim and J. K. Lee, "Capture effects of wireless CSMA/CA protocols in Rayleigh and shadow fading channels," IEEE Trans. Veh. Technol., vol. 48, no. 4, pp. 1277-1286, Jul. 1999.

[31] L. Zheng, N. Lu, and L. Cai, "Reliable wireless communication networks for demand response control," IEEE Trans. Smart Grid, vol. 4, no. 1, pp. 133-140, Mar. 2013.

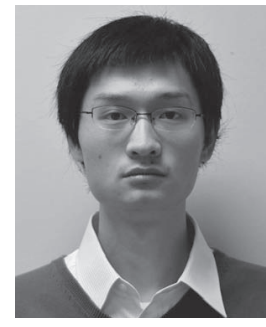

Lei Zheng (S'11) received the B.S. and M.S. degrees in electrical engineering from Beijing University of Posts and Telecommunications, Beijing, China, in 2007 and 2010, respectively. He is currently working toward the Ph.D. degree with the Department of Electrical and Computer Engineering, University of Victoria, Victoria, BC, Canada. His research interests include machine-to-machine networks, including medium access control protocol, radio resource allocation in wireless networks, and demand response control in smart grids.

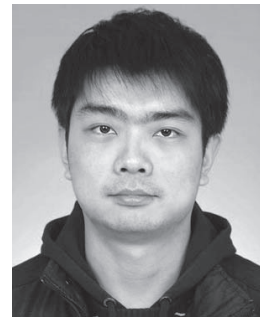

Minming Ni (S'08-M'13) received the Ph.D degree from Beijing Jiaotong University, Beijing, China, in 2013. During his Ph.D. study, he was a visiting student with McMaster University, Hamilton, ON, Canada. After his graduation, he worked as a Postdoctoral Research Fellow with the University of Victoria, Victoria, BC, Canada, with Prof. J. Pan and Prof. L. Cai. His research interests include network modeling and performance analysis, VANETs, and D2D communications.

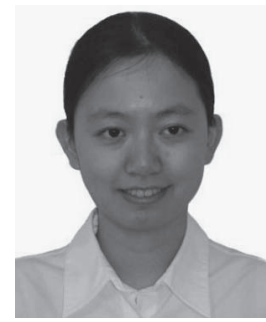

Lin Cai (S'00-M'06-SM'10) received the M.A.Sc. and Ph.D. degrees in electrical and computer engineering from the University of Waterloo, Waterloo, ON, Canada, in 2002 and 2005, respectively. Since 2005, she has been an Assistant Professor and then an Associate Professor with the Department of Electrical and Computer Engineering, University of Victoria, Victoria, BC, Canada. She has been an Associate Editor for the IEEE TRANSACTIONS ON WIRELESS COMMUNICATIONS, the IEEE TRANSACTIONS ON VEHICULAR TECHNOLOGY, the EURASIP Journal on Wireless Communications and Networking, the International Journal of Sensor Networks, and the Journal of Communications and Networks. Her research interests include wireless communications and networking, with a focus on network protocol design and control strategy supporting emerging applications in ubiquitous networks. 


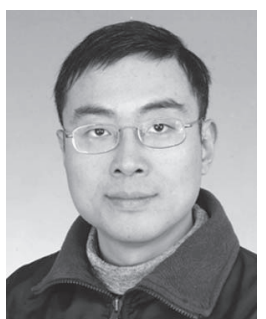

Jianping Pan (S'96-M'98-SM'08) received the Bachelor's and Ph.D. degrees in computer science from Southeast University, Nanjing, China. He did his postdoctoral research at the University of Waterloo, Waterloo, ON, Canada. He is currently an Associate Professor of computer science with the University of Victoria, Victoria, BC, Canada. He also worked at Fujitsu Labs and NTT Labs. His area of specialization is computer networks and distributed systems, and his current research interests include protocols for advanced networking, performance analysis of networked systems, and applied network security. He received the IEICE Best Paper Award in 2009, the Telecommunications Advancement Foundation's Telesys Award in 2010, the WCSP 2011 Best Paper Award, the IEEE Globecom 2011 Best Paper Award, the JSPS Invitation Fellowship in 2012, and the IEEE ICC 2013 Best Paper Award. He has been serving on the Technical Program Committee of major computer communications and networking conferences, including the IEEE INFOCOM, ICC, Globecom, WCNC, and CCNC. He is an Ad Hoc and Senor Networking Symposium Cochair of the IEEE Globecom 2012 and an Associate Editor of the IEEE Transactions on Vehicular Technology. He is a Senior Member of the ACM.

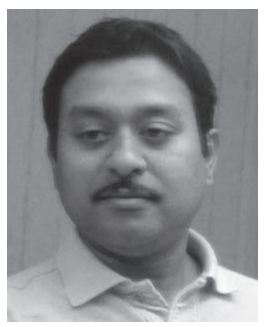

Chittabrata Ghosh did his postdoctoral research in the Electrical Engineering Department, University of Washington, Seattle, WA, USA. He is currently a Principal Researcher with the Nokia Research Center, Berkeley, CA, USA, and an Affiliate Assistant Professor with the University of Washington. He is an active contributor to IEEE 802.11ah, which is a long-range low-power amendment to the IEEE 802.11 wireless local area network standard, and was a member of the Executive Review Committee toward its draft development. His research interests include developing spectrum-sharing concepts for next-generation commercial communications systems and solving coexistence challenges among cooperating heterogeneous wireless networks. He is the author of more than 30 conference and journal publications and of over 30 filed or published patents. He has received the IEEE PACRIM Gold Award for Best Communications Paper in 2011 and the Best Paper Award at the IEEE PIMRC 2012. He is serving as an Editor for the IEEE TRANSACTIONS ON WIRELESS COMMUNICATIONS and is currently the Vice Chair of the IEEE Technical Committee on Simulations (TCSIM).

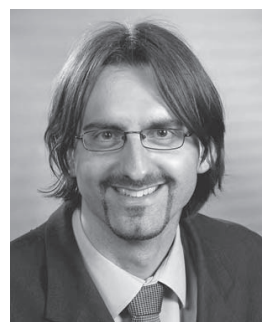

Klaus Doppler received the Ph.D. degree from Helsinki University of Technology, Helsinki, Finland, in 2010 and the M.Sc. degree in electrical engineering from Graz University of Technology, Graz, Austria, in 2003. He is heading the Radio Communications research in Nokia Technologies. His team is responsible for the 3GPP LTE, WLAN, and $5 \mathrm{G}$ research and standardization of Nokia Technologies and explores new opportunities in radio implementation. In the past, he has been leading the Wireless Systems team at the Nokia Research Center, Berkeley, CA, USA, and contributed to research activities on the design and integration of novel radio concepts into wireless systems, including device-to-device communication, (cooperative) relaying, and multiband operation. He has more than 75 pending and granted patent applications and has published 30 journal and conference publications and book chapters. He received several inventor awards at Nokia between 2007 and 2011. 